\title{
CONTRIBUIÇÕES DO PROJECT MODEL CANVAS NO GERENCIAMENTO DE CURSOS ONLINE: DO PLANEJAMENTO ÀS ETAPAS DE EXECUÇÃO
}

\author{
SÃO PAULO/SP MARÇO/2018
Tatiane Carvalho Ferreira - UNICSUL - tatiane.ferreira@cruzeirodosulvirtual.com.br
Marcos Andrei Ota - UNICSUL - marcos.ota@cruzeirodosul.edu.br \\ Tipo: Relato de Experiência Inovadora (EI) \\ Categoria: Métodos e Tecnologias \\ Setor Educacional: EDUCAÇÃO SUPERIOR, EDUCAÇÃO CORPORATIVA, EDUCAÇÃO \\ CONTINUADA EM GERAL

\begin{abstract}
RESUMO
Devido ao grande aumento de projetos nas organizações e instituições educacionais, a complexidade de cumprir todas as etapas de realização de um projeto em um mundo "versão beta" no qual as mudanças ocorrem rapidamente, tornou-se um desafio. A fim de facilitar alguns procedimentos, modelos visuais de gestão são adotados, pois contribuem com uma maior visualização dos problemas e ainda facilita o entendimento de alguns aspectos que não são bem visíveis. Partindo dessa situaçãoproblema, este artigo tem o objetivo de demonstrar a aplicação do Project Model Canvas em um curso fictício, com a finalidade de contribuir para o planejamento da produção de novos cursos online e ainda, reunir competências para o gerenciamento de outros projetos educacionais. Espera-se, que a aplicação dessa metodologia visual possa contribuir na elaboração de trabalhos futuros que desejam seguir essa vertente.
\end{abstract}

Palavras-chave: Gestão de Projeto; Project Model Canvas; Curso Online; Produção; Educação a Distância

\section{AGRADECIMENTOS}

AGRADECIMENTO A CRUZEIRO DO SUL VIRTUAL. 


\section{INTRODUÇÃO}

Com a expansão da modalidade de educação a distância (EaD) e os avanços tecnológicos na educação, o número de instituições fornecedoras de produtos voltados para a EaD está crescendo, tanto em número de novas empresas em todo o Brasil, quanto em inovações nas suas soluções (CENSOEAD, 2017). Como consequência, ocorre o aumento do número e complexidade de projetos dentro das organizações e Instituições de Ensino Superior (IES), com cursos da modalidade semipresencial ou totalmente a distância.

No entanto, pouco tem sido pesquisado a respeito do planejamento e gerenciamento dos cursos online (CASTRO e LADEIRA, 2009), sendo boa parte das pesquisas voltadas para os modelos pedagógicos e ambientes virtuais de aprendizagem (MORAN, 1994; PIMENTEL,1995; PRETI, 1996). Diante desse contexto, surge a necessidade de recorrer às outras áreas do conhecimento, como por exemplo, as contribuições advindas da gestão de projetos, seja pela aplicação de metodologias e/ou estratégias de gerenciamento.

Tendo em vista que o gerenciamento de projetos possui alguns modelos visuais de gerenciamento, como o Project Model Canvas (PMC), este estudo tem como objetivo demonstrar a aplicação do modelo visual PMC para apoiar o planejamento de cursos online. A partir disso, espera-se obter considerações confiáveis para que outros trabalhos possam ser executados nessa área de conhecimento, além de possibilitar a utilização desse modelo para o contexto de projetos educacionais.

\section{GERENCIAMENTO DE PROJETOS}

Por mais que a área de gerenciamento de projetos esteja em alta no momento, essa é uma ideia antiga, que já existe desde o lançamento do satélite Sputnik, em meio à Guerra Fria, pela União Soviética. O termo foi, cronologicamente, popularizado por Drucker, em 1954, através do termo gerenciamento de objetivos, influenciando a formação da teoria de gestão de projetos. O gerenciamento de projetos era utilizado em ações militares e foi se aperfeiçoando aos poucos até começar a ser incorporado nas grandes empresas. Nesse ponto, a gestão começou a servir para a reengenharia, a globalização, a automação dos processos, popularização dos computadores e internet (VALLE et al., 2010).

Como inúmeros projetos começaram a ser realizados por empresas, a PMI organizou o Project Management Body of Knowledge (PMBoK) - Guia do Conhecimento em 
Gerenciamento de Projetos. Segundo o próprio instituto de gestão, o guia fornece algumas diretrizes para que gestores administrem seus projetos de forma padronizada, para que o acúmulo de erros seja mínimo ou inexistente.

De forma sintetizada, o gerenciamento de projetos é a aplicação do conhecimento, habilidades, ferramentas e técnicas às atividades do projeto para atender aos seus requisitos (PMI, 2013). De acordo com esse guia, o gerenciamento do projeto pode ser realizado por meio da aplicação e integração de 47 processos, estrategicamente agrupados em cinco grupos de processos. Sendo eles: iniciação; planejamento; execução; monitoramento e controle; e encerramento.

O gerenciamento de projetos, até poucos anos, era uma prerrogativa apenas de grandes corporações; hoje é acessível às pequenas e médias empresas (PMEs), podendo ser o diferencial entre o sucesso e o fracasso (FERREIRA et al, 2016). Os autores complementam ainda, que a adoção de gerenciar projetos não pode ser feita de forma impetuosa e sem planejamento, havendo a necessidade de avaliar se o produto ou serviço permite essa forma de gestão.

Nesse contexto, o Guia do Conhecimento em Gerenciamento de Projetos (PMBoK), criado pelo $\mathrm{PMI}$, fornece algumas diretrizes, a fim de criar um padrão para 0 gerenciamento dos projetos. No entanto, mesmo com a facilidade de seguir uma série de regras, que servem como orientação, o gerente pode sofrer com obstáculos na hora de elaborar as atividades de cada etapa do projeto e cometer erros que afetarão o seu resultado final. Sendo assim, com alguns conceitos-base sobre o gerenciamento de projetos, apresenta-se a seguir o Project Model Canvas (PMC), ancorado às ideias de Finocchio Jr. (2013).

\section{MODELOS VISUAIS DE GERENCIAMENTO: CANVAS}

Com o avanço na área de gerenciamento de projetos e do número de empresas que estão fazendo uso do gerenciamento para obter maior sucesso em determinados processos, houve um aumento na complexidade desse ambiente, já que podem existir diversas dificuldades no que diz respeito ao planejamento e execução, como um todo, do projeto em questão, seja pela gama de projetos e/ou demandas que ocorrem concomitantes.

Dessa forma, alguns modelos de gestão podem melhorar a visualização de todas as etapas do projeto e mostrar com maior nitidez o que deve ser feito em cada uma delas. Esses modelos de gestão são conhecidos como modelos visuais de gerenciamento, 
sendo os explorados e estudados Project Model Canvas, Project Model Mind Map, Project Model Visual e o Life Cycle Canvas (MEDEIROS; SILVA, 2017).

Ainda de acordo com estudos do Sebrae (2013), alguns diferenciais desse tipo de modelo - o visual, são o pensamento visual, a visão sistêmica, a cocriação e a simplicidade e aplicabilidade. De forma geral, o modelo visual de um negócio e/ou projeto consiste em usar desenhos para representar ideias, compreender o todo baseando-se em análises de interação entre as partes, permitir que pessoas não envolvidas em um projeto entendam o que está sendo realizado e simplificar o processo de verificação e correção de dados. Tendo em mente algumas definições breves sobre o modelo visual de negócios, recorre-se às contribuições do Project Model Canvas (FINOCCHIO JÚNIOR, 2013) para subsidiar o planejamento de um projeto.

\subsection{Project Model Canvas}

Segundo a Project Builder (2013), o PMC utiliza conhecimentos da neurociência que propõem uma forma mais amigável de conceber um plano de projetos, respondendo às seguintes perguntas fundamentais: por quê, o quê, quem, como, quando e quanto. Além disso, o PMC contempla responder a especificações sobre o gerente de projetos (GP) e ao pitch, ou seja, uma frase que apresenta o projeto de forma sucinta (MEDEIROS; SILVA, 2017, p.6). Essa etapa pode ser simplificada pela Figura 1 abaixo:

Figura 1: Questões do Project Model Canvas

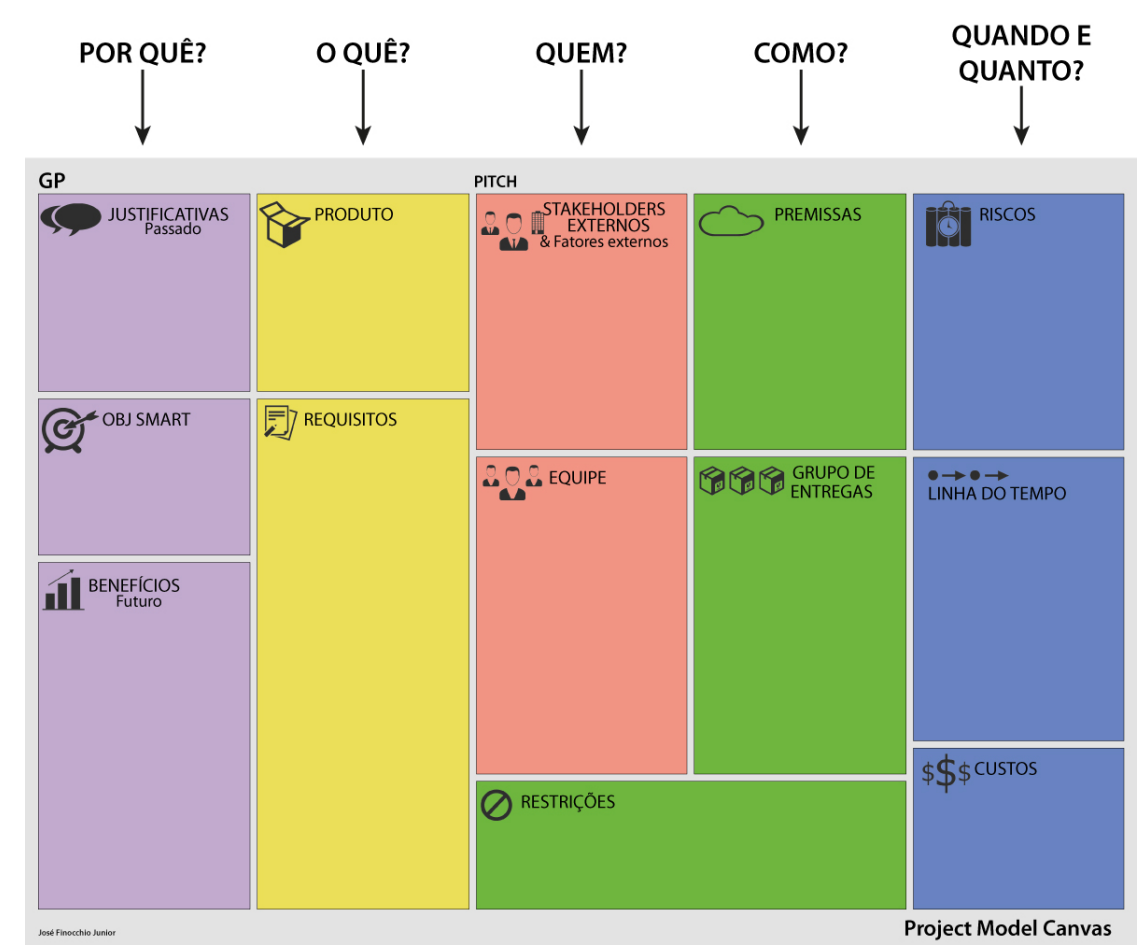


Fonte: Adaptado de Finocchio Júnior (2013)

De acordo com Finocchio Júnior (2013), para fazer uso do PMC é necessário que o plano do projeto seja criado de forma colaborativa, com a integração dos integrantes da equipe, e que, pelo menos, um membro possua conhecimentos básicos sobre a gestão de projetos.

Segundo a Project Builder[1] (2013), o primeiro passo a seguir na construção do PMC é responder ao pitch, resumindo o projeto em apenas uma frase; na sequência, é necessário colocar na seção de justificativa todos os problemas que a organização apresenta e quais são as necessidades que não estão sendo atendidas; no objetivo S.M.A.R.T, coloca-se o objetivo do projeto de maneira que fique Specific (específico), Measurable (mensurável), Attainable (atingível), Realistic (realista) e Time Bound (temporizável); na seção de benefícios, deve-se apontar o que a empresa irá conquistar após implantar determinado projeto; o produto corresponde ao resultado final do projeto, podendo ser, também, um serviço; os requisitos do quadro definem a qualidade do produto ou serviço para que exista valor para o cliente; na seção de stakeholders devem ser identificados todos os fatores externos que podem afetar o projeto; em equipe, devese listar todos os responsáveis pelas entregas do projeto; em premissas considera-se as suposições dadas como certas; em grupos de entregas quais os componentes concretos, mensuráveis e tangíveis que serão gerados pelo projeto; nas restrições, descreve-se todas as limitações do projeto; na seção de riscos, considera-se todos os eventos futuros e incertos com certa relevância; em linha do tempo define-se quando ocorrerão as entregas; e, em custos, quanto será pago para a conclusão do projeto.

\footnotetext{
O PMC também explica que não basta preencher os campos referentes aos conceitos-chave, que trata da concepção do projeto. É necessário também garantir a consistência entre os blocos, estabelecendo uma integração adequada entre as áreas preenchidas. Com a integração, é possível resolver inconsistências, indefinições e contradições junto a clientes e patrocinadores. Após estas três etapas, sugere-se compartilhar o documento produzido com os demais membros da organização e que as informações contidas neste plano de canvas possam ser transportadas para planos de projetos)formais, apresentações e outros documentos (MEDEIROS; SILVA, 2017, p.7).
}

A Figura 2, a seguir mostra o esquema do quadro que deve ser construído a partir do PMC.

Figura 2: Quadro do Project Model Canvas 


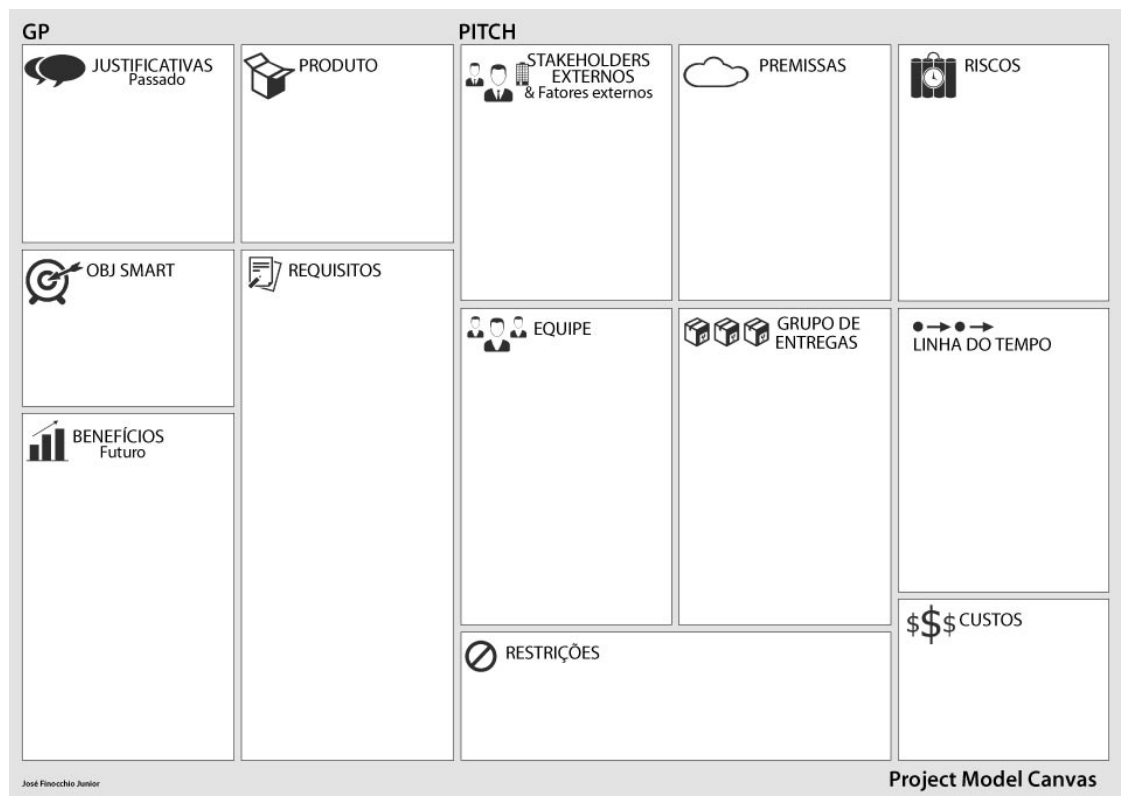

Fonte: Finocchio Júnior (2013)

Levando em consideração os conhecimentos adquiridos sobre a gestão de projetos e o Project Model Canvas, com a revisão bibliográfica realizada para este artigo, pode-se, na seção 4 deste trabalho, a fim de realizar a aplicação do PMC para o planejamento da produção de um curso online.

\section{APLICAÇÃO DO PMC PARA O PLANEJAMENTO DA PRODUÇÃO DE UM CURSO ONLINE}

Esta seção representa a aplicação do PMC para o planejamento da produção do curso fictício "Metodologia PMI aplicada no gerenciamento de projetos", utilizado como exemplo. Estão contempladas as etapas da taxonomia do PMC, conforme as contribuições de Finocchio Jr. (2013).

\subsection{Exemplo aplicado}

Na prática, cada pergunta fundamental é respondida juntamente com seus devidos componentes e na sequência definida por Finocchio Jr. Sendo assim, inicia-se definindo o gerente do projeto e o Pitch.

- GP: João da Silva.

- Pitch - Produção do Curso: Metodologia PMI aplicada no gerenciamento de projetos.

Após essa etapa, os componentes são preenchidos com post-its na sequência apresentada a seguir:

Por quê?

Justificativa: apresentar um novo modelo de curso na área de GP em que o aluno possa colocar na prática a teoria aprendida; preparar profissionais que estão em busca de adquirir práticas de gestão de projetos; modernizar o nicho mercadológico; e oferecer um modelo de curso online diferenciado. 
Objetivo do Projeto - Emprega-se os objetivos S.M.A.R.T para definir essa etapa: criar um modelo de Design Instrucional fixo para o curso online voltado à Gerenciamento de Projetos - Metodologia PMI, empregando trilha de aprendizagem e aprendizagem baseada em problemas, com oferta em 4 meses.

Benefícios do Projeto:

Define-se o grau de contribuição para cada objetivo. As intensidades das contribuições são: muito alta, alta, entre média e alta, média, entre média e baixa, baixa e muito baixa.

- utilizar metodologias ativas no curso online (alta);

- desenvolver competências e habilidades (alta);

- engajamento dos alunos (alta);

- maior visibilidade no mercado EaD (entre média e baixa); e

- formato pouco explorado (média).

\section{O quê?}

Produto: curso online em gerenciamento de projetos - metodologia PMI, focado no desenvolvimento de competências requeridas para gerir projetos com eficiência.

Requisitos:

- ambiente virtual de aprendizagem;

- trilha de aprendizagem;

- todos os recursos do curso precisam ser compatíveis com o LMS Blackboard;

- professores com, no mínimo, especialização;

- uso de tecnologias digitais;

- aprendizagem baseada em problemas;

- módulos separados por grupos de processos;

- atividades de simulação; e

- toda área de conhecimento deverá dispor de estudos de caso em que o aluno possa aprender de forma mais proativa.

\section{Quem?}

- Stakeholders externos e fatores externos:

- pessoas interessadas no curso de Gerenciamento de Projetos - Metodologia PMI;

- patrocinadores do projeto;

- professores contratados para produzir os conteúdos dos módulos.

- prazos dos conteudistas; e

- disponibilidade de recursos financeiros.

Equipe do projeto:

- autores para os módulos;

- coordenador pedagógico;

- coordenador de produção de materiais didáticos;

- coordenador de produção audiovisual;

- designer instrucional;

- designer gráfico;

- diagramador;

- assistente de produção audiovisual; e

- analista de suporte;

\section{Como?}

Premissas: reaproveitamento de conteúdos existentes para compor atividades dos módulos; terceirização caso a equipe não comporte a dimensão do projeto; e disponibilidade de duas pessoas do setor de produção audiovisual 
para auxiliar no projeto;

Grupo de entregas:

- materiais teóricos de cada módulo;

- atividades baseada em problemas para cada processo de gerenciamento de projetos;

- videoaulas;

- objetos de aprendizagem; e

- verificar materiais de reaproveitamento.

Restrições:

- o curso terá que ser ofertado dentro de 4 meses;

- a receita não poderá ultrapassar o valor da criação de um curso padrão da instituição de ensino; e

- a equipe de produção de materiais didáticos está em número reduzido de colaboradores disponíveis para o projeto comparado a quantidade de demanda que o setor recebe;

\section{Quando e quanto?}

Riscos:

Risco global baseado na tabela de Nível de Risco Global por Categoria disponível no livro PM Canvas.

Risco global alto: Destaque para as categorias: Complexidade do trabalho (alto) e envolve processos organizacionais críticos (alto).

Risco específico 1:

- Causa: fatores externos que atrapalhem os conteudistas

- Risco: autores atrasarem os conteúdos

- Efeito: atraso no material a ser disponibilizado ao aluno

- Probabilidade: 4 Impacto: 7

Risco específico 2:

- Causa: falta de mão de obra para realizar as entregas

- Risco: necessidade de aumentar a receita planejada

- Efeito: ultrapassar o gasto planejado para o curso

- Probabilidade: 3 Impacto: 4

Risco específico 3:

- Causa: demanda em constante crescimento

- Risco: aquisição de novos projetos durante a execução do atual

- Efeito: atrasos nas entregas no projeto por falta de mão de obra

- Probabilidade: 8 Impacto: 3

Linha do Tempo:

- Entrega 1: materiais teóricos de cada módulo; e verificar materiais de reaproveitamento.

- Entrega 2: videoaulas.

- Entrega 3: atividades baseadas em problemas para cada processo de gerenciamento de projetos.

- Entrega 4: objetos de aprendizagem.

Custos fictícios:

material teórico $R \$ 21.600$ 
videoaulas $R \$ 7.200$

atividades $\mathrm{R} \$ 4.000$

Diante do exemplo aplicado, tem-se no link https://goo.gl/Vo9RGp o modelo visual do curso planejado a partir das premissas do PMC. A saber, a versão digital foi extraída do site http://pmcanvas.com.br/ onde há disponibilização gratuita do template para download. Dado o contexto de produção de cursos online, acredita-se que ao realizar a aplicação no formato digital, possibilita compartilhar o que fora planejado com todos os envolvidos e suas respectivas atribuições entre outros componentes fundamentais.

\section{ALGUMAS CONSIDERAÇÕES}

Inicialmente, tem-se que o gerenciamento de projetos começou a ser necessário nas empresas dado que muitos projetos precisavam de uma certa organização, caso contrário. estariam fadados ao insucesso. Com o grande aumento dos projetos nas organizações, surgiram os modelos visuais de gerenciamento, que buscavam facilitar o processo de gestão.

Com base no objetivo proposto por este trabalho, este artigo buscou cumpri-lo de forma a levantar referências acerca do gerenciamento de projetos, do modelo visual de gerenciamento - PMC - para então, demonstrar a aplicação no contexto de projetos educacionais, evidenciados a partir de um curso fictício. Com o PMC, realizado na forma de um quadro, os componentes são preenchidos e podem ser facilmente associados ao planejamento de produção de um curso online, desde que esse seja visto como um projeto. Através do PMC e conhecendo quais os critérios que devem ser levados em consideração para a construção desse modelo, pode-se dividir o planejamento de criação de um curso a distância nas seguintes etapas: por quê, o quê, quem, como, quando e quanto. Ou seja, correlaciona-se as etapas do PMC com a produção do curso e responde-se as questões dadas para que o projeto obtenha maior sucesso na sua conclusão.

Tendo o planejamento e a criação de um curso online, o Project Model Canvas é capaz de averiguar tanto a questão de gestão, que envolve o planejamento, quanto a de produção de conteúdo, que envolve tanto a criação do design quanto a do material didático. Sendo assim, o estudo realizado por este artigo considera que o PMC é um importante modelo visual de gerenciamento que pode auxiliar no desenvolvimento da produção de cursos online, visto que é capaz de pontuar melhor as etapas de gerenciamento, propiciando o sucesso total do projeto. Em suma, o gerenciamento de projetos configura-se como uma oportunidade de reunir contribuições para o contexto da 
educação online, uma vez que poderá minimizar os riscos negativos acerca da qualidade de um determinado curso produzido.

\section{REFERÊNCIAS}

ABED - Associação Brasileira de Educação a Distância. Censo EAD.BR: relatório analítico da aprendizagem a distância no Brasil 2016. Curitiba: InterSaberes, 2017

CASTRO, J. M.; LADEIRA, E. S. Gestão e planejamento de cursos a distância no Brasil. Revista Gestão e Planejamento, Salvador, v. 10, n. 2, p. 229-247, jul./dez. 2009.

FERREIRA, Maurício et al. Gerenciamento de projetos. 2016.

FINOCCHIO JÚNIOR, J. Project Model Canvas: gerenciamento de projetos sem burocracia. Rio de Janeiro: Elsevier/Campus, 2013.

MEDEIROS, Bruno Campelo; SILVA, Rafael Rodrigues da. Gestão Visual em Projetos: Analisando os modelos de canvas à luz do Guia PMBOK. Anais do VI SINGEP. São Paulo, Brasil, 13 e 14/11/2017.

MORAN, José Manuel. Interferências dos meios de comunicação no nosso conhecimento. Revista Brasileira de Comunicação, São Paulo, v. 17, n. 2, jul./dez, 1994.

PIMENTEL, Nara. O ensino a distância na formação de professores. Revista Perspectiva, Florianópolis, n. 24, 1995.

PMI. Um Guia do Conhecimento em Gerenciamento de Projetos (Guia PMBOK), 2013.

PRETI, Oreste. Educação a Distância: uma prática educativa mediadora e mediatizada. In: PRETI, Oreste. (Org.). Educação a distância: inícios e indícios de um percurso. Cuiabá: NEAD/IE - UFMT, 1996.

PROJECT BUILDER. Guia definitivo do Project Model Canvas. 2017. Disponível em: Acesso em 10 jan. 2017.

SEBRAE. O quadro de modelo de negócios: um caminho para criar, recriar e inovar em modelos de negócios. 2013.

STRINGHETTA, Maria do Carmo Teles Ferreira; ALMEIDA, Siderly do Carmo Dahle de. Gestão do conhecimento na preparação e produção de materiais para cursos online. Maringá, 2015.

VALLE, A. B. do et al. Fundamentos do Gerenciamento de Projetos. Rio de Janeiro: Editora FGV, 2010. 